\title{
Virtual Machining Accuracy Verification by Triangular Patch Comparison
}

\author{
Liwen Guan ${ }^{1, a}$, Jianwei Xiong ${ }^{2, b}$ \\ ${ }^{1}$ Department of Mechanical Engineering, Tsinghua University, Beijing 100084, China \\ ${ }^{2}$ School of Mechatroics Engineering, University of Electronic Science and Technology of China, \\ Chengdu 611731, China
}

aguanlw@tsinghua.edu.cn, b337945058@qq.com

\begin{abstract}
Keywords: Virtual manufacturing, Machining accuracy verification, Triangle patch.
Abstract. Machining accuracy verification is an important method in virtual manufacturing to determine if desired machining accuracy can be achieved before actual machining. A machining accuracy verification method is proposed in this paper. The design part model in STereo Lithography(STL) format is imported and converted into discrete model with new triangle patches according to the discrete result of the machined part. The machining error is treated as the distance and normal projection of the distance between central points of the corresponding triangle patches on the machined part and the design part. The simulation result shows the efficiency of the proposed method.
\end{abstract}

\section{Introduction}

Machining accuracy verification is one of the major steps of virtual manufacturing, which calculates the error of virtual machined part, analyzes and corrects potential error in Numerical Control(NC) code before actual machining so as to achieve desired machining accuracy.

There are two types of simulation systems: solid modelling based and curved surface modelling based. In solid modelling based simulation system, there are two accuracy verification methods: solid model boolean operation and discrete model boolean operation. Voelcker and Hunt[1] proposed a Numerical Control(NC) simulation and accuracy verification method based on Constructive Solid Geometry(CSG) solid model, in which virtual machining is performed by boolean operation of solid models. In curved surface modelling based simulation system, there are three accuracy verification methods: vector cutting method, vector accuracy verification method with local subdivision, and $\mathrm{Z} \& \mathrm{~N}[2-3]$ method, in which the virtual machining error is obtained by calculating the distance of discrete points of machined part and desired work part in $\mathrm{Z}$ direction or in normal vector direction.

In this paper, we adapt curved surface modelling based simulation system. The design part model in STL format is imported and converted into a new triangle patch model according to the discrete result of the machined part. A machining accuracy verification method is proposed in which the machining error is calculated with distance $d$ of central point of corresponding triangle patch on machined part and design part, and normal projection of distance $d$.

\section{Machining accuracy verification algorithm}

The machining accuracy verification is a quantitative error analysis by comparison of the virtual machining workpiece and the design part, which purpose is to investigate whether the accuracy of virtual machining is satisfied with the design requirement. The virtual machining simulation system developed in this paper uses the discrete triangle method to establish the rough stock model. The cutting process is realized by calculating the intersection height of discrete points along $\mathrm{Z}$ axis and the tool swept volume, then rebuilding the triangles to achieve dynamic cutting. So the simulation precision is affected by triangle discrete precision. The calculating error of the intersection height is also the main source of simulation error. Because the surface is discretized into small triangles, calculating error at discrete points along $\mathrm{Z}$ axis is quite different from the actual error when curvature is large, the triangle patch error should be calculated along $\mathrm{Z}$ axis with the projection along normal direction of the triangle of the error at discrete points. The main steps of the accuracy verification algorithm is as following: 
(1) According to the desired accuracy, determine the discretization precision of rough surface, discretize the rough surface into discrete points, connect the discrete points into uniform triangle small planes, obtain the triangular mesh model;

(2) The $\mathrm{Z}$ vector of the surface at each discrete point intersects with tool swept. After obtained simulation result, update triangle mesh of work piece and get the machined work piece model;

(3) Input the STL model file of the design model, and the triangular mesh model is built according to the accuracy of the discrete model of rough stock;

(4) Calculate the of $\mathrm{Z}$ difference of discrete point and the design model, and the $\mathrm{Z}$ error is obtained. Then the $\mathrm{Z}$ error index of the discrete points is set up;

(5) Calculate the $\mathrm{Z}$ error value at the center point of each discrete triangle, and project the $\mathrm{Z}$ error to the normal vector of the triangular surface of the part, obtain the error between triangular surface and the design part, then set up error index set of triangular patch;

(6) Show the error distribution in the 3D graphics window with cloud graph, and error distribution data with data sheet.

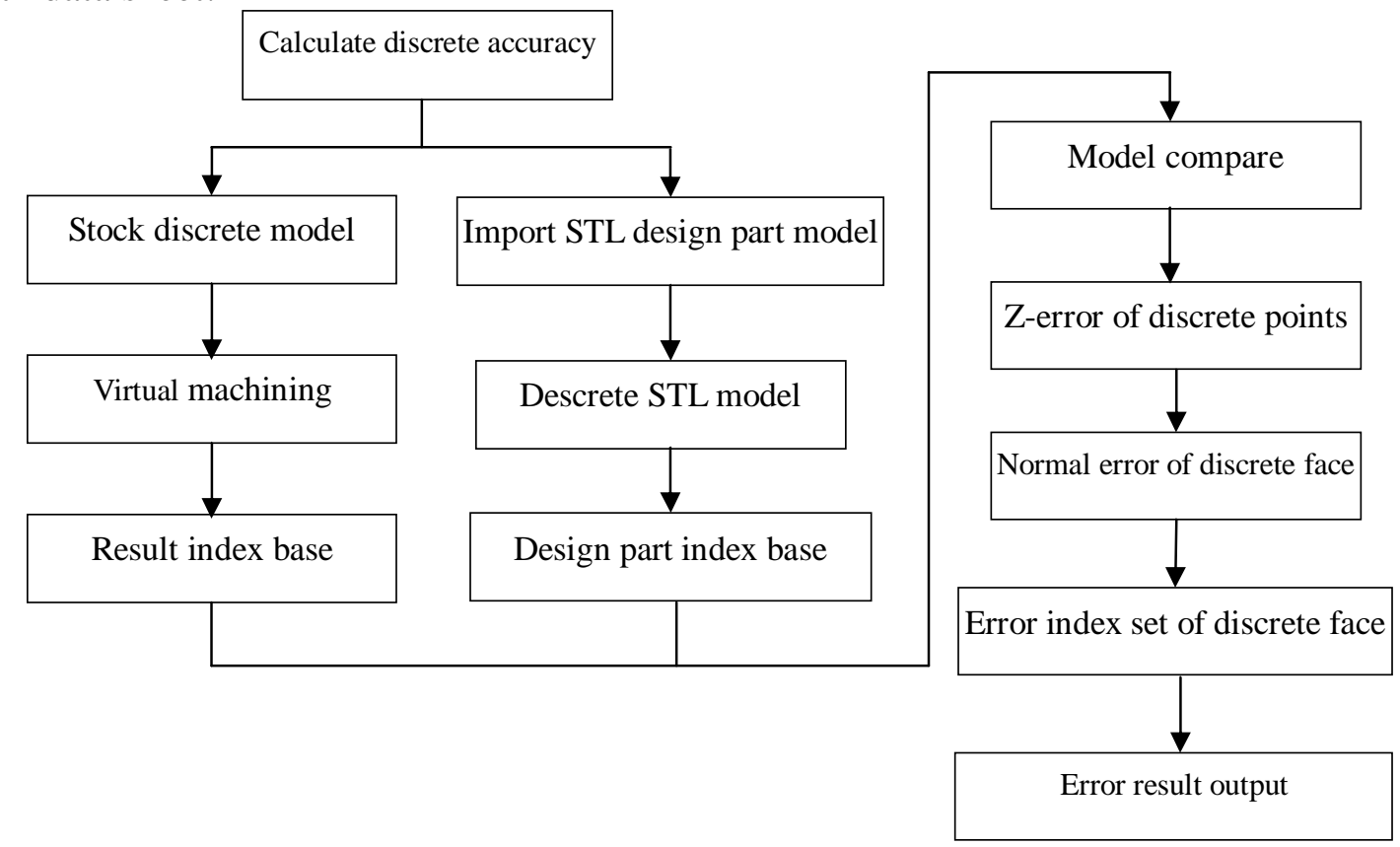

Fig.1 Machining accuracy verification workflow

\section{Discrete model of rough stock}

In the simulation system of numerical control processing in this paper, rough stock model is established by triangles from uniform discrete points. Machining accuracy is calculated by comparing discrete points and design model, so the discrete accuracy of rough stock has a great impact on the calculating result. It is shown that the discrete error of rough stock is composed of surface approximation error $e_{s}[7]$ and sampling error $e_{p}$ of discrete point. To determine the distance between discrete points, the surface approximation error and the sampling error of discrete points are analyzed.

Surface approximation error $e_{s}$ is due to using triangular patches to approximate the actual surface when the surface is discretized. The accurate calculation of surface approximation error is quite complex, so an approximate calculation method is described in this paper.

As shown in Fig.2, $R_{1}$ is the minimum radius of curved surface, $R_{x}$ and $R_{y}$ are distance between discrete points in $x$ and $y$ direction. The approximate error of the surface is :

$$
\begin{aligned}
& e_{s}=R_{1}-\sqrt{R_{1}^{2}-\left(\frac{\sqrt{2}}{2} R_{x}\right)^{2}} \\
& R_{x}=\sqrt{2 R_{1}^{2}-2\left(R_{1}-e_{s}\right)^{2}}=\sqrt{4 R_{1} e_{s}-2 e_{z}^{2}} \approx 2 \sqrt{R_{1} e_{z}}
\end{aligned}
$$



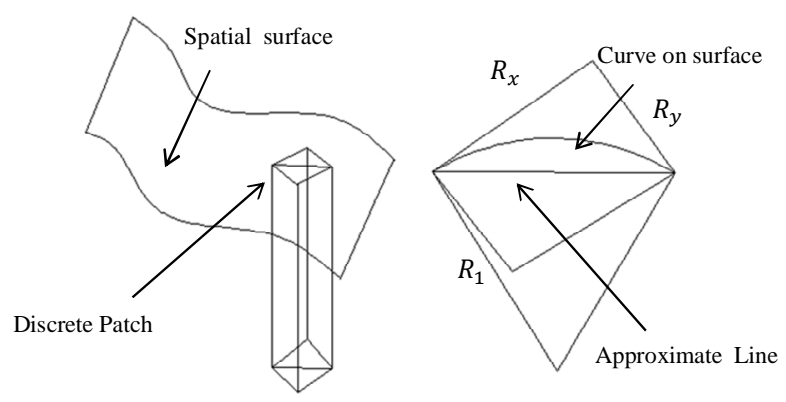

Fig.2 Approximate error

Due to the discreteness of points, the cutter can only change the ray height at discrete points, other gaps is filled by triangular patches instead of cutting surface, so the sampling error is generated. As shown in Fig. 3[8]. If select the intersection point of the cutter and the surface as sampling point, supporting all sampling points are satisfied with accuracy requirement, the error of points on the surface which are not the sampling point is $e_{p}$. Assign cutting tool radius is $r$, the distance between discrete points is:

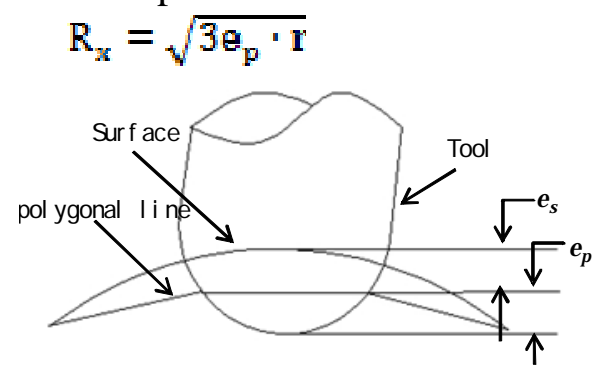

a)

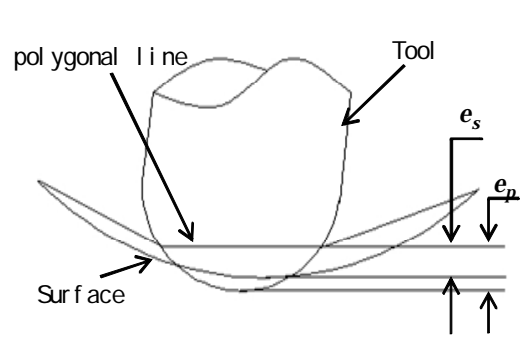

b)

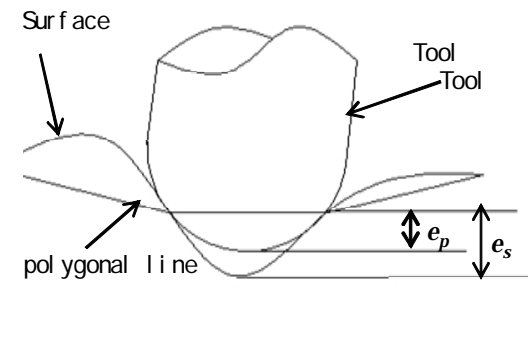

c)

Fig.3 Approximation error and sampling error

When discrete part surface is convex, the total error of discrete surface is the sum of approximation error $e_{s}$ and sampling error $e_{p}$ as shown in Fig. 3a. When discrete part surface is concave, the total error of discrete surface is the maximum of approximation error $e_{s}$ and sampling error $e_{p}$ as shown in Fig. 3b. To concave part with total error threshold $e$ :

$$
\mathrm{e}=\mathrm{e}_{\mathrm{g}}, \mathrm{e}=\mathrm{e}_{\mathrm{p}}
$$

The distance $R_{x}$ of discrete points is the minimum of values derived from equation (3). If the rough part has convex and concave surface, as shown in Fig. 3c, the discrete distance is the sum of approximation error $e_{s}$ and sampling error $e_{p}$.

\section{Discrete model of design part}

Usually the design part is modelled by commercial CAD software. To general purpose the design part is converted into STereo Lithography (STL) format which is imported into the simulation system. So machining accuracy verification is to compare the machining result and the STL model. The discrete points on machined part are projected on the design part surface along $\mathrm{Z}$ direction. So the machining error is the distance between discrete point and the project point.

For convenience of comparison, the design part model should be discretized according to discrete points on the machined part, and the triangular prisms are obtained from the corresponding discrete triangle patches on the machined part and the design part. The discrete triangular prism intersects the machined part and the design part with two triangles. The problem of error calculation is converted into calculation the distance between two triangles. 

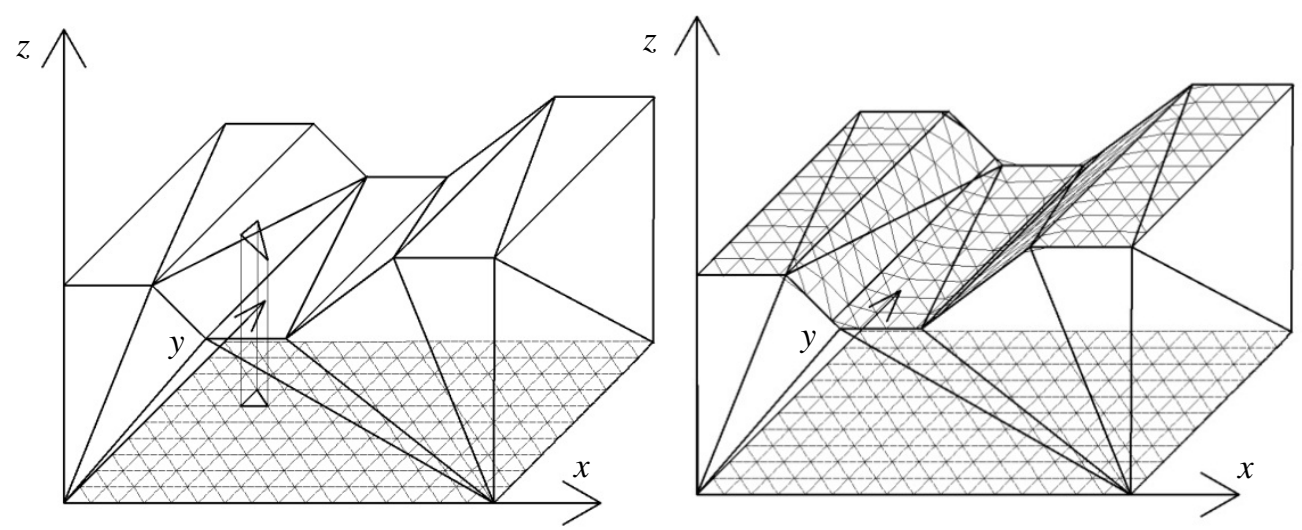

a) before discretion b) after discretion

Fig. 4 Discrete model of design part

\section{Machining error calculation}

The calculation of error between the two discrete faces includes two steps: distance calculation of the central points of two discrete faces, and the distance projection calculation along the normal vector. The distance of the central points along $\mathrm{Z}$ axis is the distance average of three corresponding points of the discrete triangle patches. As shown in Fig., triangle ABC is on design part surface, and $\mathrm{A} " \mathrm{~B} " \mathrm{C}$ " is on the machined part surface. The central distance $d$ along $\mathrm{Z}$ axis is:

$$
d=\frac{1}{3}\left[\left(\mathrm{Z}_{\mathrm{A} r r}-\mathrm{Z}_{\mathrm{A}}\right)+\left(\mathrm{Z}_{\mathrm{E} r}-\mathrm{Z}_{\mathrm{E}}\right)+\left(\mathrm{Z}_{\mathrm{Cr} r}-\mathrm{Z}_{\mathrm{C}}\right)\right]
$$
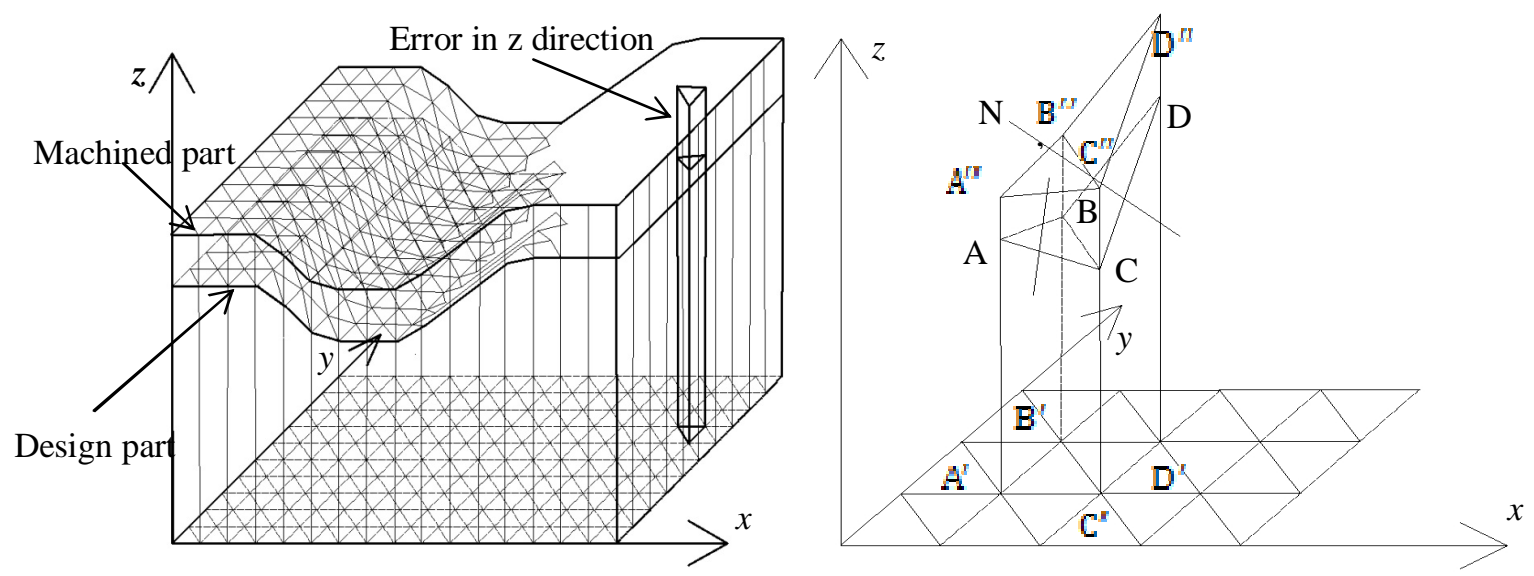

Fig.5 Discrete comparision between design part and machined part

The normal vector of the triangle patch on the design part is:

$$
\vec{N}=\overrightarrow{A C} \times \overrightarrow{A B}
$$

After virtual machining, and calculating machining error by comparing machined work part and design part, paint each discrete triangle face according to error value, bright red, dark red, violet and dark blue indicates seriously over-cut, slight over-cut, slight under-cut and seriously under-cut respectively. As shown in Fig.5, the error data can be outputted into data file which can be browsed by user.

Supposed that the unit vector along $Z$ axis is $\vec{Z}$, the discrete error $\mu$ between triangle paths on the machined part and the design part is:

$$
\mu=\frac{\vec{N} \cdot \vec{z}}{|\vec{N}|} d
$$

After virtual machining, and calculating machining error by comparing machined work part and design part, paint each discrete triangle face according to error value, bright red, dark red, violet and dark blue indicates seriously over-cut, slight over-cut, slight under-cut and seriously under-cut respectively. As shown in Fig. 6, the error data can be outputted into data file which can be browsed by user. 


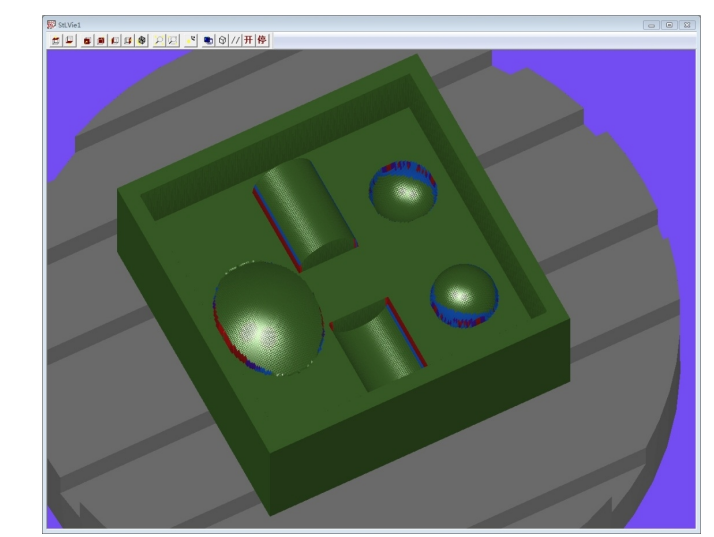

Fig. 6 Error cloud figure of accuracy verification

\section{Summary}

A machining accuracy verification method is proposed based on triangle patch comparison. Firstly, the algorithm for accuracy verification is introduced. Then the discrete method of the machined and design part is described. Finally, the calculation method is given. The simulation result shows the efficiency of the proposed method.

\section{Acknowledgement}

This research was financially supported by the Major National S\&T Program (2013ZX04001-021).

\section{References}

[1] Hunt WA, Veolcker HB. Post simulation error analysis of automatic verification of programs for numerically controlled machine tools. Production Automation Project Tech Memo No 34, University of Rochester (January 1982).

[2] Jeard RB, Hussaini SZ and Drysdale RL. Approximate method for simulation and verification of application numberically controlled machining programs. The Visual Computer. 5(1989) 329-348.

[3] $\mathrm{Hu}$ XL. Accuracy verification algorithm in NC virtual manufacturing technique. Mechanical Design and Manufacturing. 3(2008) 96-97.

[4] Wu FZ.Research of changing the direction in NC simulation machining process.CAM and NC machining.8(2001) 36-37.

[5] Zhao. NC graphic machining simulation based on image space.The Chinese Mechanical Engineering.9(1998) 28-31.

[6] Gossard DC. Application of set theory to the verification of NC tapes. Proc North American Metalworking Conf (April 1978).

[7] Chen YL. Research on key techniques in five-axis NC machining geometry simulation. Northwestern Polytechnical University (2004).

[8] Mou X, Liu XY, Zhang RM. Error detection and dynamic simulation for free surface machining. Journal of Huazhong University of Technology. 23(1995) 64-66. 